\title{
Physical Exercise to Improve the Balance of the Elderly with the Risk of Falling: a Literature Review
}

\author{
Dwi Nur Anggraeni, Arlina Dewi \\ University of Muhammadiyah Yogyakarta, Indonesia \\ dewieanggraeny94@gmail.com
}

\begin{abstract}
Most falling occurs in the elderly because of the decrease of balance and muscle strength. Physical training for elderly needs to conduct to enhance physical fitness of the elderly and it can reduce the risk of falls by increasing muscle strength by increasing body balance in the elderly. The aim of this research is to find out the effectiveness of physical exercise to improve balance in the elderly. Method to collect database is conducted in Google Scholar and Pubmed to find literature. Literature used was literature that required the inclusion criteria published in the last three years, then the result is chose and the review is conducted. Result: there are 6 literature to be reviewed. Based on literature review, it is found that respondents are 65-91 years old. Most respondents are elderly women. Type of physical exercise as follow, Visual Feedback, Tai Chi Chuan, Whole Body Vibration, Balance Focus Exercise, Progressive Resistive Exercise, Telepresence. Falling risk in elderly can be decreased by applying regular physical exercise in the elderly. Beside, physical exercise can improve muscle ability, maintain balance, reduce anxiety, and reduce fear of falling. Elderly also able to do daily activities well.
\end{abstract}

Keywords: Balance, Elderly, Physical Exercise, Risk Of Falling

Received July, 25, 2019; Revised August 24, 2019; Accepted September 13, 2019

(c) (7) (2) STRADA Jurnal Ilmiah Kesehatan, its website, and the articles published there in are licensed under a Creative Commons Attribution-ShareAlike 4.0 International License. 


\section{STRADA Jurnal Ilmiah Kesehatan}

DOI: $10.30994 /$ sjik.v8i2.217

ISSN: 2252-3847 (print); 2614-350X (online)

Vol.8 No.2. November 2019. Page.108-116

\section{BACKGROUND}

Falling is the main cause of fatal and non-fatal injury in elderly. Nearly a third of the population above aged 65 falls every year, half of them falling into injury. Aging is accompanied by an overall reduction in mobility and a decrease in sensory integration which has been linked to falls (Anson et al., 2018).

United Nation estimates that the aging population growth rate in the 21 st century will be much higher than in the previous century. According to estimates of the Population Reference Bureau, the world population reached 7.1 billion in mid-2012, with $8 \%$ over the age of 65 . In particular, the proportion of the aging population in developed countries is as high as $16 \%$, indicating that the world has become an aging society (Tseng et al., 2016). In 2006, Center for Disease Control and Prevention reported that falling incident in elderly above the age of 65 were $30 \%$, whereas elderly above the age of 80 were $50 \%$. Autumn can cause many problems for the elderly, and falling is one of the 5 causes of death in the elderly. Preventing of falling in the elderly is currently an important problem. One important factor which causes falling in the elderly is the stability of poor posture. It is caused by aging which causes a gradual decrease in sensory function, central processing, motor control of muscle strength, and nerve pathways. Balance damage can also be an important factor that causes falling (Tseng et al., 2016).

Physical function decrease as long as the age increases, especially in the elderly. Falling is a result of a decrease in physical fitness, especially in muscle strength and strength itself. Early detection of falling risk factors and appropriate falling prevention are considered as an effective method to avoid bad experiences related to falling such as fractures. It is because falling risk factors are not striking for people who have not experienced falls, most fall prevention studies focus on a one-time experience or recurring experience, not those who do not have a history of falls (Zhao, Chung, \& Tong, 2017). Balance loss has been shown to be the highest cause of falls in this population and, therefore, an important problem which must be addressed to prevent falls and provide confidence to carry out daily activities (ADL) in a safe manner. Balance is also an important predictive factor for functional recovery (Yousefian Molla, Sadeghi, \& Kahlaee, 2017).

Physical exercise has been proven effective in decreasing risk and falling incident frequency/level (Hong, Kong, \& Yoon, 2018). This physical exercise aims to obtain physical fitness. Physical fitness in the elderly is needed so that it can improve balance and the elderly can carry out normal daily activities safely and independently without undue fatigue. Functional fitness consists of the basic physical capacity needed to maintain the flexibility of an active and independent lifestyle, muscle strength, agility and balance, and endurance (Zhao et al., 2017).

\section{OBJECTIVE}

This study aimed to find out the effectiveness of physical exercise to improve balance in the elderly.

\section{METHODS}

\section{Search Method}

The inclusion criteria is used to choose literature review study such as: full text (journals can be downloaded), human (the object of research used is the elderly aged 65-91), the type of research study RCT (Randomized Control Trial) and journals of the last 3 years. Whereas 


\section{STRADA Jurnal Ilmiah Kesehatan}

DOI: $10.30994 /$ sjik.v8i2.217

ISSN: 2252-3847 (print); 2614-350X (online)

Vol.8 No.2. November 2019. Page.108-116

the exclusion criteria were not included because in the journal obtained has met these criteria.

\section{Selection Criteria}

Searching the literature was conducted in December 2018. the researcher carried out data source using some databases such as PubMed and Google Scholar. Key words used were fall risk", "fall prevalence", "fall status"," fall incident", " exercise", " stretch", "physic activity", "training", "balance test", "stability test", " elderly". Researcher gave limitation to search for journals related to physical exercise to improve balance in the elderly. In accordance with the keywords and inclusion criteria which have been included, there are 30 journals from Pubmed and 114 from Google Scholar.

\section{Search Outcome}

After Literature search in Pubmed and Google Scholar, then it was conducted review of abstracts that matched the objectives of Pubmedof 15 and from Google Scholar of 5. Furthermore, full text was reviewed and results obtained from Pubmed were 8 and from Google Scholar were 2. Then extraction was done for the final results. literature in the last 3 years so that the data obtained is valid and up-to-date, the results are Pubmed 4 and from Google Scholar 2. The final results of database searches in Pubmed and Google Scholar are 6 literature.

Table 1. Characteristic Study

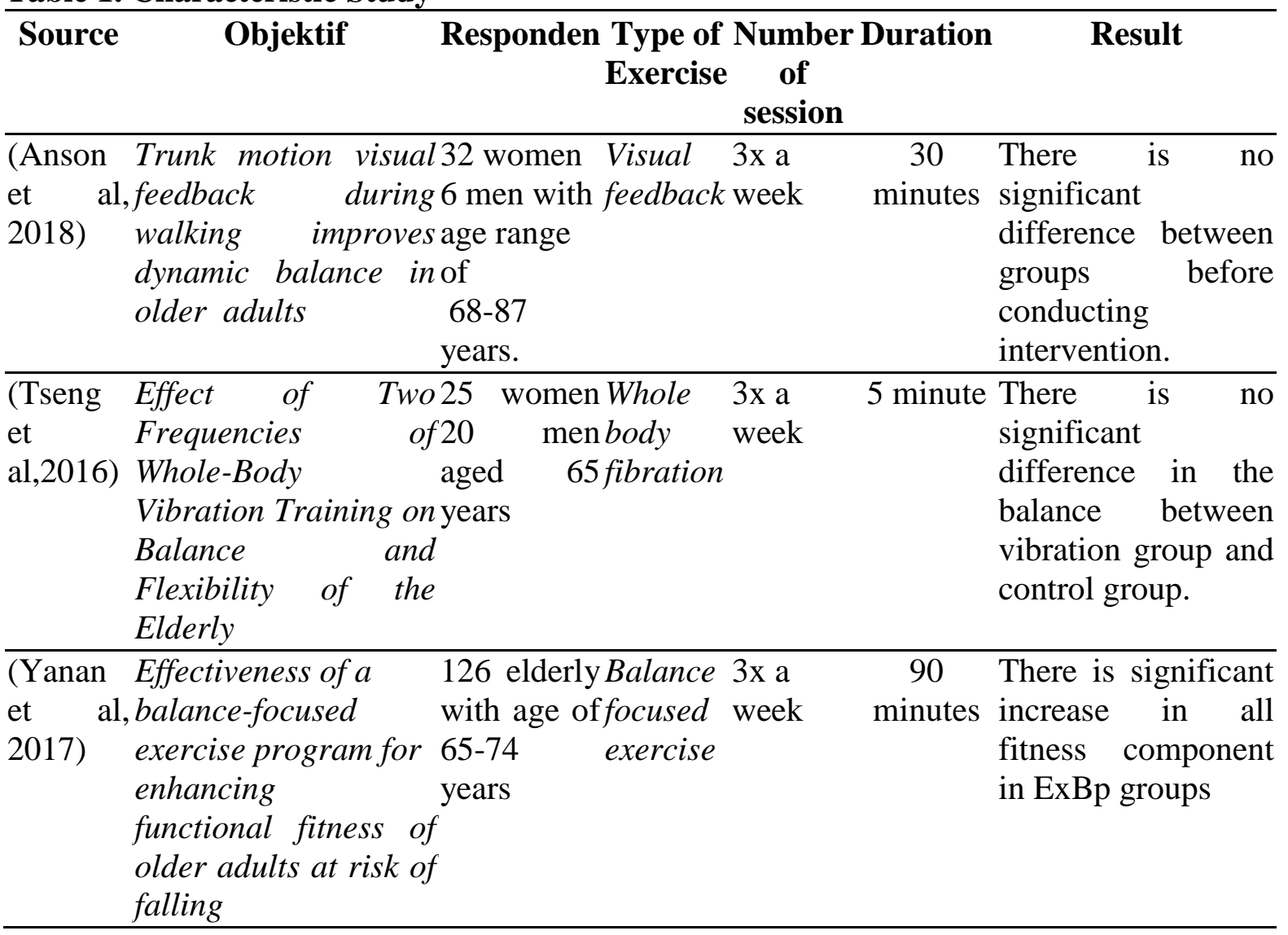




\section{STRADA Jurnal Ilmiah Kesehatan}

DOI: $10.30994 /$ sjik.v8i2.217

(Lida et Tai Chi Chuan can 33 women Tai Chi2x a al, 2018) improve balance and 27 men age Chuan week reduce fear of falling of $\quad 60-80$ in community years dwelling older adults

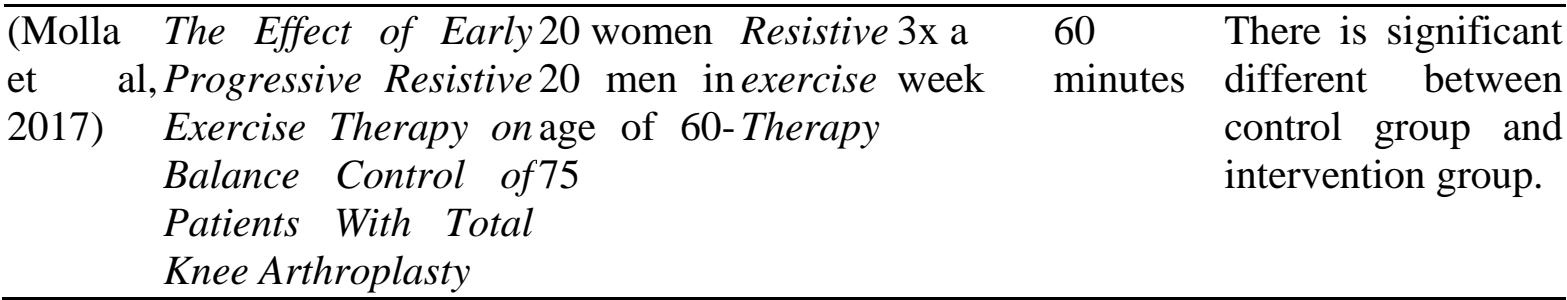

55 menit There is no difference in control group and intervention group.

\section{RESULTS}

\section{Respondent}

From 6 reviewed journals, 4 research journals states that the number women elderly respondents are more than men elderly respondents in 32 types of physical visual feedback exercise from 38 respondents (Anson et al., 2018). Whole body fibration are 25 of 45 respondents (Tseng et al., 2016), Tai Chi Chuan are 33 of 60 respondents (Hosseini et al., 2018), Telepresence exercise are 34 of 34 respondents (Hong et al., 2018). A journal mentions the number of respondents of 126 elderly but it does not explain their gender specifications in physical exercise Balance focused exercise (Zhao et al., 2017), then another journal explains the same number of female and male respondents, namely 20 respondents in Resistive exercise Therapy (Yousefian Molla et al., 2017).

\section{Type of Physical Exercise}

Types of physical exercise which are used to improve balance as follow:

1. Visual Feed Back is a balance training tool to reduce the risk of falling. This technology provides a new way to improve balance capabilities in a safe, controlled and attractive environment. Most VFB training is based on leg movements. Training with VFB trunk motion while walking on a treadmill will improve balance as measured by a balance clinical test. The VFB training session lasts 30 minutes and it is carried out 3 times a week for 4 weeks. Subjects are instructed to use handrails only if they lose balance. Each session, the subject's "comfortable speed" is determined according to the speed of the treadmill being increased until the subject says "too fast" and then decreases the speed until the subject says "too slow". The process is repeated for 30 minutes resulting in 8 12 battles running per session. VFB sequence randomly during each training session, every 2 minutes walking the researcher gives a VFB translation or orientation. Experimental groups are told about the type of VFB before every 2 minutes of battle. The control group also attended the same training schedule. The procedure is the same except that they do not receive training on how to use VFB, and they do not see or interact with VFB. However, to walk and sit at rest is the same as the experimental group (Anson et al., 2018).

2. Whole Body Vibration is vibration of all body which is well-known as forms of exercise using a vibration platform to produce regular up and down movements. Vibration is distributed from the bottom of the foot throughout the body to trigger receptors in the 


\section{STRADA Jurnal Ilmiah Kesehatan}

DOI: $10.30994 /$ sjik.v8i2.217

body to produce an adaptive response and further improve balance. The respondents who met the inclusion criteria are randomly assigned to three groups: high frequency vibration training group $(40 \mathrm{~Hz})$, low frequency vibration group $(20 \mathrm{~Hz})$, and no vibration control group $(0 \mathrm{~Hz})$. The instrument used in this study is a whole body vibration training machine (Commercial Grade Vibration Machine LV-1000; X-trend, Taiwan). The vibration pattern is a peak-to-peak amplitude of $4 \mathrm{~mm}$, and with a frequency of $20 \mathrm{~Hz}$ and $40 \mathrm{~Hz}$ and the peak acceleration produced is $1.5 \mathrm{~g}$ and $5.5 \mathrm{~g}$ respectively, for 5 minutes each time. Vibration training lasts for 3 months, during which the subject receives a 5 minute whole body vibration training session three times per week. Subjects stand on a vibration platform with knees slightly bent (Tseng et al., 2016).

3. Exercise for Balance Improvement Program (ExBP) is exercise developed to improve balance and prevent the falling for people at risk of falling. ExBP is an interesting and appropriate exercise which is suitable for the elderly to do in daily activities. This program can significantly improve dynamic balance and static balance with a sensation that is compromised by using laboratory test equipment. ExBP focuses on eight aspects of exercise, as follow: 1) center mass control; 2) muscle strength in the lower limbs (especially for ankle dorsiflexion and knee extension); 3) range of motion (especially in the ankle joint); 4) plantar tactile sensitivity and joints around the knee and ankle; 5) gait patterns, agility, and response time; 6) balance control; 7) focus on balance; and 8) confidence in carrying out tasks to improve balance. Participants in the control group did not receive care. Except that ExBP and TC exercises, all participants are asked to maintain their usual lifestyle. If there are changes in their daily lives, then the habit is reported (eg, physical activity, quality of sleep, diet, and medication), participants are instructed to keep a diary and report to research assistants without involvement in other research procedures each Sunday. Two physical trainers for seniors with qualification certificates run two intervention programs separately. Both interventions are all carried out in a series of 90 minute training sessions, with 3 sessions per week, for 16 weeks, in addition to an 8-week follow-up. Each training session consisted of 15 minutes warmup, 25 minutes of exercise, 10 minutes of rest, another 25 minutes of exercise, and 15 minutes of cooling. Exercise intensity is monitored using the South Korean version of the Borg 6-20 Rating of the Perceived Exertion (RPE) scale. 18 The average intensity of training through 16 weeks training ranges from RPE 11 (mild) to RPE 13 (rather hard). The training place is located in the community, which is quite close to where the participants live. The average attendance rate for participants was $92.1 \%$ for the ExBP group and $88.5 \%$ for the TC group (Zhao et al., 2017).

4. Tai Chi Chuan is exercise program which includes balance exercise to decrease falling risk. Tai chi Chuan includes a series of strength training and balance training, proven effective in preventing falls. Tai Chi Chuan is originally developed as a martial art in China and it has often been used as an exercise to improve mental or physical health in young and old populations. In this study, researcher interviewed people at the local retirement association and explained the purpose of the study. Participants who meet the inclusion criteria and agreed to participate in this study filled out written informed consent. In the first session, we assess balance in all participants. The participants in the intervention group performed a Tai Chi Chuan exercise style for eight weeks, twice a week, and 55 minutes per session. Each session consists of 5 minutes warm-up, 35 minutes Tai Chi Chuan exercise, and 5 minutes cooling. All program is managed by Tai 


\section{STRADA Jurnal Ilmiah Kesehatan}

DOI: $10.30994 /$ sjik.v8i2.217

ISSN: 2252-3847 (print); 2614-350X (online)

Vol.8 No.2. November 2019. Page.108-116

Chi Chuan expert trainers accompanied by a researcher (LH) in an outdoor location in a community park. The control group does not receive any intervention. At the end of the study, all participants are reassessed for balance and fear of falling. Finally, after the intervention period, the control group participants are referred to participate in the Tai Chi Chuan program. Tai Chi Chuan consists of a series of movements such as dance, slow and continuous. This movement emphasizes joint rotation, coordination, and gradual narrowing of the position of the lower extremities. Tai Chi Chuan exercises are characterized by balancing body, mind, breathing and emotions and making the muscles of the body flexible and vibrant (Hosseini et al., 2018).

5. Resistive Exercise Program is physical exercise to increase load and excess in extensor muscles to build muscle mass and strength. All patients receive instructions regarding the use of appropriate tools for ambulation. Continuous passive movement starting on the first postoperative day for the range $0^{\circ}$ to $60^{\circ}$ is increased according to tolerance. The rehabilitation programs of the two groups become different during the second week of the outpatient rehabilitation protocol. The control group performed open kinetic chain exercises (not holding the load) and the kinetic chain is closed (holding the load) initially with 2 sets of 10 repetitions, then developed into 3 sets of 10 repetitions. Strengthening exercises consist of isometric contraction QC, sitting knee extension (not holding), straight leg lifting, side-lying hip abduction, and standing with a hamstring. Weight training consists of terminal knee extension standing, single leg position, and sliding against the wall. This Latihamn is done 3 times a week for 60 minutes. Exercises are as follows: (1) Static cycling with a minimum voltage of 5 minutes with a weekly increase in duration of up to 30 minutes: resistance to pedals increases with patient tolerance; (2) up and down stairs: the exercise will begin with stairs 2 to 4 in. Furthermore, go up to the higher stairs (up to 6-8 in) with increased strength during the study rehabilitation phase; and (3) load flexion and knee extension. Weight is increased to maintain the maximum intensity level targeted at 10 repetitions, the maximum weight used for strengthening exercises is $4.5 \mathrm{~kg}$ ankle weight. The duration of cycling exercise, ladder height, and the amount of endurance gradually increased on a weekly basis and they are determined by the patient's tolerance not to cause pain and swelling persistence (longer than Mouse et al., 2017).

6. Telepresence Exercise Program is program which is conducted at home as a new intervention method to prevent falling in high risk falling elderly. This exercise develops a new telepresence training platform using information and communication technology to provide supervised physical training sessions to elderly participants at home, with real-time feedback from remote instructors trained to assess the physical, cognitive, and emotional responses of aged participants. go on. Fall-related risk factors were assessed personally 1 week earlier to begin intervention. In addition, at that time, each participant and instructor of the sport was given a tablet PC (personal computer), folding chairs, sports mats, resistance bands, and instructions explaining the simple steps needed to turn on and operate the tablet, facilitating participation in the program web-based telepresence training. During the intervention period, nutrition guidance and sports education are given to all participants every 4 weeks. During the 12-week intervention period, participants are encouraged to maintain the same level of physical activity and calorie intake as before participating in this study. During the education session, we instructed participants to inform instructors who held educational sessions on changes in levels of physical activity or nutritional intake. Posttest assessment is carried out 


\section{STRADA Jurnal Ilmiah Kesehatan}

DOI: $10.30994 /$ sjik.v8i2.217

ISSN: 2252-3847 (print); 2614-350X (online)

Vol.8 No.2. November 2019. Page.108-116

during the last week of the 12-week program. The level of physical activity and CG nutritional intake were examined every 2 weeks by telephone and during the education session. Web Technology Real-Time Communication (WebRTC) allows telepresence training platforms to make real-time voice calls, video chat and text messages in browsers without additional software (Hong et al., 2018).

\section{The Effect of Physical Exercise}

The effects of physical exercise to improve the balance are following:

(Tseng et al., 2016) suggests that exercise effect of vibration in all body for elderly during test is each of the eight targets is given direction. Subjects are randomly directed to move their center of gravity to the touch target and then return to the center. The formula is (Actual distance traveled) / (Straight line distance to target) 100\%, and unit is percentage (100\%). Higher values indicate better control of the movement of the body's center of gravity and better balance. This balance scoring system has been verified, and that reliability and validity is acceptable. (Zhao et al., 2017) reveals that the overall results of this study shows that participants who receive a 16-week ExBP training can obtain a substantial increase in functional fitness. Significant and moderate differences between the ExBP and CON groups in CS, UG, and Step at posttest suggest that ExBP 16 weeks can produce a notable increase in lower limb muscle strength, agility and balance, and aerobic endurance. The insignificant changes between the posttest test and follow-up were found in terms of the AC, CS, SR, UG, and Step tests, indicating that ExBP training had an effect on muscle strength, lower body flexibility, agility and balance, and aerobic endurance from the group the target can be maintained at least 8 weeks immediately after the intervention. (Anson et al., 2018) explains that elderly with balance issue can improve their dynamic balance after exercising using VFB treadmill movement. Individuals with poorer sensory functions can obtain benefit more from VFB movement while walking than individuals with intact sensory function. Overall, four weeks of training with trunk motion VFB on a running treadmill results in a significant increase in balance and mobility as measured by the BEST and mBEST. (Hosseini et al., 2018) reveals that Tai Chi Chuan effectively improves balance and fear will fall and it can be considered a practical and useful method for prevention of falls in the elderly who live in the community. Tai chi chuan exercises can improve the balance of the elderly who live in the community. Tai Chi Chuan has a positive effect on balance, gait and quality of life in the elderly. Several factors contribute to balance disorders during the aging process, including nerve conduction speed, muscle flexibility, and the development of osteoarthritis. (Yousefian Molla et al., 2017) our results reveal that a more profound increase in instatic, semidynamic, and dynamic balance may arise when progressive resistive exercise can be added to routine physical therapy at the beginning of the second postoperative week. One mechanism that may explain the increase induced by isotonic resistive exercise in balance is an increase in muscle strength and endurance. (Hong et al., 2018) suggests that telepresence training programs have a positive effect on risk factors associated with falls in the elderly who live in a community of women at high risk of falls. Telepresence technology for exercises that can be conducted at home can function as an effective intervention to improve the physical and psychological factors associated with falls.

\section{DISCUSSION}

An important factor causing falling in elderly is poor posture stability, caused by a gradual decrease in sensory function, muscle strength, motor control, and nerve pathways. Balance 


\section{STRADA Jurnal Ilmiah Kesehatan}

DOI: $10.30994 /$ sjik.v8i2.217

ISSN: 2252-3847 (print); 2614-350X (online)

Vol.8 No.2. November 2019. Page.108-116

damage can also be an important factor causing a decrease. Many studies show that exercise can improve the balance of performance of the elderly. Aerobic dance can improve posture control of the elderly to improve their balance. Whole body vibrations are becoming increasingly popular as a form of exercise that uses a vibration platform to produce regular up and down movements. Several studies have included whole body vibration exercise in treatment protocols for patients with stroke, multiple sclerosis, and chronic obstructive pulmonary disease (Tseng et al., 2016). Elderly people with balance problems can improve balance by walking dynamically on their land after a VFB (visual feed back) trunk exercise on a treadmill runs. Elderly people with vestibular loss or sensory disorders can take more benefit from VFB exercise than individuals with normal sensory function. The effect of training with trunk motion VFB may be more beneficial for individuals with more severe balance disorders. Considering the benefits of improved VFB training for balance, it can be a useful training strategy for improving balance. This technology provides new breakthroughs to improve balance capabilities in a safe, controlled and interesting environment (Anson et al., 2018).

Considering the relation between balance and other fitness component, exercise program focusing on balance can be useful to improve other fitness components. Therefore, it can improve all functional fitness. Previous studies are mostly conducted using general exercises (eg, Tai Chi). There are consistent results regarding increased balance (Zhao et al., 2017). Several studies have shown that Tai Chi Chuan is effective in preventing falling and improving balance, muscle strength, proprioception, and endurance in the elderly. However, several studies show that 12 months of practicing Tai Chi Chuan does not change the risk of falling in elderly who live at home. Tai Chi Chuan remains controversial, therefore further studies are needed to explain the benefits of the Tai Chi Chuan program in the elderly (Hosseini et al., 2018). Special training programs for the elderly are needed. Exercise for a balance enhancement program is a newly developed exercise that aims to improve balance and prevent falling for those who do not fall who are at risk of falling. This test determines that a balance training program is an interesting and appropriate exercise that is suitable for elderly people to do in their daily lives (Zhao et al., 2017).

\section{CONCLUSION}

From 6 journals, 4 journals explain that there are relations between physical exercise used by improving balance in elderly. Physical exercises are Whole body fibration, Balance focused exercise, Resistive Exercise Exercise, Telepresence exercise. While the two journals that have no relationship are physical exercise using Visual feedback and Tai Chi Chuan.

In the Tai Chi Chuan research journal, researchers reveal that this study do not conduct follow-up to evaluate the risk of falls after the intervention no longer reported long-term impact. It is because participants and researchers distinguish their group allocations, and the generalization of findings is limited. Participants are limited to the elderly who live in one condition and they are influenced by their cultural environment, economic situation, and social settings (Hosseini et al., 2018).

In research with Visual Feed Back intervention, it shows that recruitment of research subjects based on reported balance problems may result bias sample. It can't be generalized to clinical population. Many subjects were not classified as subjects who have experienced a fall and scored above the threshold to identify an increased risk of falls. It is not known if there are individuals who experience changes in sensory function or sensitivity after training that might contribute to the results of this study. The results show an increased effect for 


\section{STRADA Jurnal Ilmiah Kesehatan}

DOI: $10.30994 /$ sjik.v8i2.217

individuals with greater sensory impairment is preliminary, but it can help determining which patients are suitable for this type of intervention in clinical settings. It is not known how long the beneficial effects of VFB balance training last.

\section{REFERENCES}

Anson, E., Ma, L., Meetam, T., Thompson, E., Rathore, R., Dean, V., \& Jeka, J. (2018). Trunk motion visual feedback during walking improves dynamic balance in older adults: Assessor blinded randomized controlled trial. Gait and Posture, 62(March), 342-348. https://doi.org/10.1016/j.gaitpost.2018.03.044.

Hong, J., Kong, H.-J., \& Yoon, H.-J. (2018). Web-Based Telepresence Exercise Program for Community-Dwelling Elderly Women With a High Risk of Falling: Randomized Controlled Trial. JMIR MHealth and UHealth, 6(5), e132. https://doi.org/10.2196/mhealth.9563.

Hosseini, L., Kargozar, E., Sharifi, F., Negarandeh, R., Memari, A.-H., \& Navab, E. (2018). Tai Chi Chuan can improve balance and reduce fear of falling in community dwelling older adults: a randomized control trial, 14(6), 1024-1031. https://doi.org/10.12965/jer.1836488.244.

Tseng, S., Y., Hsu, P., S., Lai, C., L., Liao, W., C., Lee, M., C., \& Wang, C., H. (2016). Effect of Two Frequencies of Whole-Body Vibration Training on Balance and Flexibility of the Elderly: A Randomized Controlled Trial. American Journal of Physical Medicine and Rehabilitation, 95(10), 730-737. https://doi.org/10.1097/PHM.0000000000000477.

Yousefian Molla, R., Sadeghi, H., \& Kahlaee, A., H. (2017). The Effect of Early Progressive Resistive Exercise Therapy on Balance Control of Patients with Total Knee Arthroplasty. Topics in Geriatric Rehabilitation, 33(4), 286-294. https://doi.org/10.1097/TGR.0000000000000165.

Zhao, Y., Chung, P., K., \& Tong, T., K. (2017). Effectiveness of a balance-focused exercise program for enhancing functional fitness of older adults at risk of falling: A randomised controlled trial. Geriatric Nursing, 38(6), 491-497. https://doi.org/10.1016/j.gerinurse.2017.02.011 